Finite-size effects on the phase transition in the three-dimensional Gross-Neveu model

This content has been downloaded from IOPscience. Please scroll down to see the full text.

2012 EPL 9711002

(http://iopscience.iop.org/0295-5075/97/1/11002)

View the table of contents for this issue, or go to the journal homepage for more

Download details:

IP Address: 200.130.19.138

This content was downloaded on 27/08/2014 at 11:59

Please note that terms and conditions apply. 


\title{
Finite-size effects on the phase transition in the three-dimensional Gross-Neveu model
}

\author{
F. C. Khanna ${ }^{1,2(a)}$, A. P. C. Malbouisson ${ }^{3(b)}$, J. M. C. Malbouisson ${ }^{4,5(c)}$ and A. E. Santana ${ }^{6(d)}$ \\ ${ }^{1}$ Department of Physics, Theoretical Physics Institute, University of Alberta - Edmonton, Alberta, T6G 2J1, Canada \\ 2 TRIUMF - 4004, Wesbrook Mall, Vancouver, British Columbia, V6T 2A3, Canada \\ ${ }^{3}$ Centro Brasileiro de Pesquisas Físicas, MCT - 22290-180, Rio de Janeiro, RJ, Brazil \\ ${ }^{4}$ Instituto de Física, Universidade Federal da Bahia - 40210-340, Salvador, BA, Brazil \\ ${ }^{5}$ Departamento de Física, Faculdade de Ciências, Universidade do Porto - 4169-007, Porto, Portugal \\ ${ }^{6}$ Instituto de Física, International Centre for Condensed Matter Physics, Universidade de Brasília \\ 70.910-900, Brasília, DF, Brazil
}

received 27 September 2011; accepted in final form 28 November 2011

published online 3 January 2012

PACS 11.10.Kk - Field theories in dimensions other than four

PACS 11.30.Qc - Spontaneous and radiative symmetry breaking

PACS 11.10.Wx - Finite-temperature field theory

\begin{abstract}
We discuss finite-size effects on the phase transition in the two-component, massive, three-dimensional Gross-Neveu model. From an analysis of four-point function and from the existence of a stable infrared fixed point of the beta-function, we get indications of the existence of a second-order phase transition. Using a generalized Matsubara prescription and zeta-function regularization techniques, we determine the dependence of the critical temperature on the size of the system.
\end{abstract}

Copyright (c) EPLA, 2012

Introduction. - The Gross-Neveu (GN) model [1], as an effective approach for quantum chromodynamics (QCD), has been useful in describing aspects of hadronic matter. These include asymptotical freedom and investigations of the continuous and discrete chiral symmetry, which are associated with the confinement/ desconfinement phase transition [2-11]. In addition, the model is also applied to condensed-matter systems. This is the case of the graphene, a honeycomb lattice with two Bravais triangular sublattices. The Hamiltonian of this structure is mapped in the $(2+1)$ GN Hamiltonian, such that the $S U(4)$ chiral symmetry, arising from the arrangement of spins, can be broken into $S U(2) \times S U(2)$, where parity and time reversal invariance are preserved. This chiral symmetry breaking is the counterpart of a quantum phase transition from the semi-metallic phase to a gapped Mott insulator [12-19]. Such a breaking of symmetry, that can be restored by raising the temperature, leads to a gap in the energy spectrum, that is of interest for electronic devices [20].

Due to these aspects, the model has been taken as a paradigmatic formalism for analyzing the phase

\footnotetext{
(a) E-mail: fkhanna@ualberta.ca

(b) E-mail: adolfo@cbpf .br

(c) E-mail: jmalboui@ufba.br

(d) E-mail: asantana@unb.br
}

transitions in quantum field theory for fermions. This in turn has been a sufficient motivation for searching analytical results for the GN model; in particular, for the physical case with $N=1$, including size-effect, where lattice calculation is a hard task $[21,22]$.

In this article we generalize a previous work [23], dealing with phase transition in the tri-dimensional GrossNeveu (GN) model. We consider the model at finite temperature, $T$, under a constraint, specified by the compactification of a spatial coordinate. This means that the system is confined in a strip of width $L$. Then the physical parameters are functions of both $T$ and $L$, and are obtained using methods already employed in several cases $[24,25]$. In previous publications $[8,9]$, spatial confinement and thermal deconfinement have been investigated, within the framework of the $N$-component GN model in the large- $N$ limit. Distinctly, here, as in ref. [23], we deal with the one-component version of the GN model. We adopt the perspective of determining a transition temperature and to investigate how this temperature is affected by the finite size of the system. This has similarities with recent articles, where finite-size effects on the phase structure of four-fermion interacting models have been considered [26-28].

It should be remarked that, distinctly from the statistical mechanics for a system of non-relativistic particles 
where sharp finite-temperature phase transitions only exist in the thermodynamic limit, fields in spaces with compactified dimensions or finite size still have an infinite (non-enumerable) number of degrees of freedom which allows the existence of sharp phase transitions; besides that, surface modes in finite-size systems provide additional continuous degrees of freedom which intervene in the symmetry-breaking aspects of the system [29].

The massive GN model in dimension $D=3$ has been shown to exist and constructed in the large- $N$ limit [30], even if, for dimensions greater than two, the model is perturbatively non-renormalizable. This is justified by observing that perturbative renormalizability is not an absolute criterion for an effective theory to be physically consistent. This fact has been used for long in phenomenological studies in particle physics [31-33]. We consider the GN model as an effective theory for QCD. In the spirit of effective theories, we can take an arbitrary coupling constant (not necessarily small) at zero temperature in the absence of spatial boundaries and we can evaluate both the $T$ - and $L$-dependent mass and coupling constant at one-loop order.

A further justification of this approximation is given by a non-perturbative analysis of the four-point function, by summing the chains of loop diagrams. This provides a non-perturbative relationship among the four-point function, temperature and the size of the system, associated to a singularity. This is a basic feature in situations theoretically close to the one we examine in this article: the linear response theory and the BCS field theoretical approach to the superconducting phase transition [34]. In these cases, the singularity of the leading contribution to the four-point function with zero-external momenta is given by the sum of all chains of one-loop diagrams. This is related to the susceptibility, which diverges at a critical temperature. It is then argued that, if a singularity of the four-point function exists, it indicates the existence of a phase transition. But in Euclidean field theories, imaginary time (inverse temperature) and spatial coordinates are on the same footing. This means that we can transpose the argument used in the linear response and BCS theories to the present situation. The difference is that now, instead of just temperature, we have both temperature, $T$, and the compactification length, $L$, as driving parameters of the transition. In other words, if the four-point function is singular for a pair of values $(T, L)$, the system of size $L$ undergoes a phase transition at the temperature $T_{c}(L)$. This will be our main concern in this note: to investigate how the the critical temperature is affected by the finite size of the system.

The model. - We consider the massive GN model in a $D$-dimensional Euclidian manifold, $\mathbb{R}^{D}$, described by the Hamiltonian

$$
\begin{aligned}
H= & \int \mathrm{d}^{D} x\left\{\psi^{\dagger}(x)\left(i \gamma^{j} \partial_{j}-m_{0}\right) \psi(x)\right. \\
& \left.+\frac{\lambda_{0}}{2}\left[\psi^{\dagger}(x) \psi(x)\right]^{2}\right\},
\end{aligned}
$$

where $m_{0}$ and $\lambda_{0}$ are, respectively, the physical zerotemperature mass and coupling constant in the absence of boundaries. The $\gamma$-matrices are elements of the Clifford algebra and we use natural units, $\hbar=c=k_{B}=1$. This Hamiltonian is obtained using standard conventions for Euclidian field theories.

Let us introduce thermal and boundary corrections to $m_{0}$ and to $\lambda_{0}$, and define the temperature and boundarydependent mass and coupling constant, $m(T, L)$ and $g(T, L)$ respectively, by

$$
m(T, L)=m_{0}+\Sigma(T, L)
$$

and

$$
g(T, L)=\lambda_{0}\left[1+\lambda_{0} \Pi(T, L)\right] .
$$

Then from eq. (1), we may write down a free-energy density of the Ginzburg-Landau type [35],

$$
\mathcal{F}=a-m(T, L) \phi^{2}(x)+g(T, L) \phi^{4}(x),
$$

where $\phi(x)=\sqrt{\left\langle\psi^{\dagger}(x) \psi(x)\right\rangle}$, with $\langle\cdot\rangle$ meaning a thermal average. The minus sign for the mass in eq. (4) implies that, in the disordered phase we have $m(T, L)<0$ and for the ordered phase $m(T, L)>0$, consistently. A secondorder phase transition occurs in the region where $m(T, L)$ changes sign from negative to positive, characterizing a spontaneous symmetry breaking. In this formalism, the quantity $\phi(x)$ plays the role of the order parameter for the transition.

As we have observed in the Introduction, we argue that at criticality, the leading contribution to the four-point function with zero-external momenta is given by the sum of all chains of one-loop diagrams. This non-perturbative calculation leads to an expression of the form

$$
\Gamma^{(4)}\left(\lambda_{0}, T, L\right)=\frac{\lambda_{0}}{\left[1-\lambda_{0} \Pi(T, L)\right]} .
$$

In our case the first two terms of the expansion in powers of $\lambda_{0}$ of such a function are given in eq. (3). The existence of a singularity of the four-point function in eq. (5) indicates a phase transition [34]; in other words if there are values of $T$ and $L$ that solve the equation,

$$
1-\lambda_{0} \Pi(T, L)=0
$$

then there is a phase transition. The nature of the transition is obtained by a study of the free energy, eq. (4). Actually, we will find later on from an analysis of the infrared fixed-point structure, that the transition is of second order and that the critical temperature is determined by the condition $m(T, L)=0$. For a given value of $L$, this leads to a $L$-dependent critical temperature $T_{c}(L)$.

Thermal and boundary correction to the mass. - We evaluate the thermal and boundary dependent self-energy, $\Sigma(D ; T, L)$, by using a generalized Matsubara formalism [24]. The Cartesian coordinates are specified by $\mathbf{r}=\left(x^{0}, x^{1}, \mathbf{x}\right)$, where $\mathbf{x}$ is a $(D-2)$-dimensional vector. The conjugate momentum of $\mathbf{r}$ is denoted by $\mathbf{k}=\left(k_{0}, k_{1}, \mathbf{q}\right)$, q being a $(D-2)$-dimensional vector in 
momentum space. The Feynman rules are modified by the generalized Matsubara prescription which changes the integration over the energy and the momentum associated with the compactified spatial dimension into sums,

$$
\begin{array}{ll}
\int \frac{\mathrm{d} k_{0}}{2 \pi} \rightarrow \frac{1}{\beta} \sum_{n_{1}=-\infty}^{+\infty}, & k_{0} \rightarrow \frac{2 \pi\left(n_{1}+\frac{1}{2}\right)}{\beta} \equiv \omega_{n_{1}}, \\
\int \frac{\mathrm{d} k_{1}}{2 \pi} \rightarrow \frac{1}{L} \sum_{n_{2}=-\infty}^{+\infty}, & k_{1} \rightarrow \frac{2 \pi\left(n_{2}+\frac{1}{2}\right)}{L} \equiv \omega_{n_{2}},
\end{array}
$$

$\omega_{n_{1}}$ and $\omega_{n_{2}}$ being Matsubara frequencies and $\beta=T^{-1}$.

At the one-loop level, the self-energy is given by $\left.\Sigma(D ; \beta, L ; s)\right|_{s=1}$, where

$$
\begin{aligned}
& \Sigma(D ; \beta, L ; s)=\lambda_{0} \frac{m_{0}}{\beta L} \sum_{n_{1}, n_{2}=-\infty}^{\infty} \\
& \times \int \frac{\mathrm{d}^{D-2} k}{(2 \pi)^{D-2}} \frac{1}{\left(\mathbf{k}^{2}+\omega_{n_{1}}^{2}+\omega_{n_{2}}^{2}+m_{0}^{2}\right)^{s}} .
\end{aligned}
$$

In order to use the dimensional regularization procedure, we introduce dimensionless quantities, $a_{1}=\left(m_{0} \beta\right)^{-2}, \quad a_{2}=\left(m_{0} L\right)^{-2}, \quad q_{j}=k_{j} / 2 \pi m_{0}, \quad$ for $j=3, \ldots, D, \omega_{n_{i}}^{\prime}=\omega_{n_{i}} / 2 \pi m_{0}$, for $i=1,2$, and $c=1 / 2 \pi$. Then we get,

$$
\begin{aligned}
& \Sigma\left(D ; a_{1}, a_{2} ; s\right)=\lambda_{0} m_{0} \sqrt{a_{1} a_{2}} \sum_{n_{1}, n_{2}=-\infty}^{\infty} \\
& \times \int \frac{\mathrm{d}^{D-2} q}{(2 \pi)^{D-2}} \frac{1}{\left(\mathbf{q}^{2}+\omega_{n_{1}}^{\prime 2}+\omega_{n_{2}}^{\prime 2}+c^{2}\right)^{s}} .
\end{aligned}
$$

After dimensional regularization, we obtain

$$
\begin{aligned}
\Sigma\left(D ; a_{1}, a_{2} ; s\right)= & \frac{m_{0}^{1-2 \nu} \lambda_{0} \Gamma(\nu)}{(4 \pi)^{(D-2) / 2} \Gamma(s)} \sqrt{a_{1} a_{2}} \\
& \times \sum_{n_{1}, n_{2}=-\infty}^{\infty}\left(\omega_{n_{1}}^{\prime 2}+\omega_{n_{2}}^{\prime 2}+c^{2}\right)^{-\nu},
\end{aligned}
$$

where $\nu=s-(D-2) / 2$.

The sum in eq. (8) is cast in the general form,

$$
\begin{aligned}
& \sum_{n_{1}, n_{2}=-\infty}^{+\infty}\left[a_{1}\left(n_{1}+\frac{1}{2}\right)^{2}+a_{2}\left(n_{2}+\frac{1}{2}\right)^{2}+c^{2}\right]^{-\nu}= \\
& 4^{\nu}\left[Z_{2}^{4 c^{2}}\left(\nu, a_{1}, a_{2}\right)-Z_{1}^{c^{2}}\left(\nu, a_{1}\right)-Z_{1}^{c^{2}}\left(\nu, a_{2}\right)\right. \\
& \left.+Z_{2}^{c^{2}}\left(\nu, 4 a_{1}, 4 a_{2}\right)\right]
\end{aligned}
$$

where

$$
Z_{1}^{b^{2}}(\nu, \alpha)=\sum_{n=-\infty}^{+\infty}\left[\alpha n^{2}+b^{2}\right]^{-\nu}
$$

and

$$
Z_{2}^{b^{2}}\left(\nu, \alpha_{1}, \alpha_{2}\right)=\sum_{n_{1}, n_{2}=-\infty}^{+\infty}\left[\alpha_{1} n_{1}^{2}+\alpha_{2} n_{2}^{2}+b^{2}\right]^{-\nu}
$$

are, respectively, the one- and two-dimensional generalized Epstein zeta-functions [36] defined for $\operatorname{Re}(\nu)>1 / 2$.
We then analytically continue $Z_{1}^{b^{2}}(\nu, \alpha)$ and $Z_{2}^{b^{2}}\left(\nu, \alpha_{1}, \alpha_{2}\right)$ to the whole complex $\nu$-plane, leading to $[8,9,36]$

$$
\begin{aligned}
Z_{1}^{b^{2}}(\nu, \alpha)= & \frac{2^{\nu} \pi^{2 \nu-1}}{\sqrt{\alpha} \Gamma(\nu)}\left[2^{\nu-2} \frac{\Gamma(\nu-1)}{(2 \pi b)^{2 \nu-2}}\right. \\
& \left.+2 \sum_{n=1}^{\infty}\left(\frac{n}{2 \pi b \sqrt{\alpha}}\right)^{\nu-1} K_{\nu-1}\left(\frac{2 \pi b n}{\sqrt{\alpha}}\right)\right]
\end{aligned}
$$

and

$$
\begin{aligned}
& Z_{2}^{b^{2}}\left(\nu, \alpha_{1}, \alpha_{2}\right)=\frac{2^{\nu} \pi^{2 \nu-1}}{\sqrt{\alpha_{1} \alpha_{2}} \Gamma(\nu)}\left[2^{\nu-2} \frac{\Gamma(\nu-1)}{(2 \pi b)^{2 \nu-2}}\right. \\
& +2 \sum_{n_{1}=1}^{\infty}\left(\frac{n_{1}}{2 \pi b \sqrt{\alpha_{1}}}\right)^{\nu-1} K_{\nu-1}\left(\frac{2 \pi b n_{1}}{\sqrt{\alpha_{1}}}\right) \\
& +2 \sum_{n_{2}=1}^{\infty}\left(\frac{n_{2}}{2 \pi b \sqrt{\alpha_{2}}}\right)^{\nu-1} K_{\nu-1}\left(\frac{2 \pi b n_{2}}{\sqrt{\alpha_{2}}}\right) \\
& +4 \sum_{n_{1}, n_{2}=1}^{\infty}\left(\frac{1}{2 \pi b} \sqrt{\frac{n_{1}^{2}}{\alpha_{1}}+\frac{n_{2}^{2}}{\alpha_{2}}}\right)^{\nu-1} \\
& \left.\times K_{\nu-1}\left(\sqrt{\frac{n_{1}^{2}}{\alpha_{1}}+\frac{n_{2}^{2}}{\alpha_{2}}}\right)\right]
\end{aligned}
$$

where $K_{\alpha}(x)$ is the Bessel function of the third kind. As a consequence, $\Sigma\left(D ; a_{1}, a_{2} ; s\right)$ reads

$$
\begin{aligned}
\Sigma\left(D ; a_{1}, a_{2} ; s\right)= & \frac{m_{0}^{1-2 \nu} \lambda_{0} \Gamma(\nu) 4^{\nu}}{(4 \pi)^{(D-2) / 2} \Gamma(s)} \sqrt{a_{1} a_{2}} \\
& \times\left[Z_{2}^{4 c^{2}}\left(\nu, a_{1}, a_{2}\right)-Z_{1}^{c^{2}}\left(\nu, a_{1}\right)\right. \\
& \left.-Z_{1}^{c^{2}}\left(\nu, a_{2}\right)+Z_{2}^{c^{2}}\left(\nu, 4 a_{1}, 4 a_{2}\right)\right] .
\end{aligned}
$$

The first term inside the square brackets in eqs. (12) and (13) (polar terms) is singular for some dimensions. We subtract them in all cases.

For $s=1$ and $D=3$, we have $\nu=s-(D-2) / 2=1 / 2$. After subtracting the polar terms and using the formulas

$$
K_{ \pm 1 / 2}(z)=\frac{\sqrt{\pi} e^{-z}}{\sqrt{2 z}}
$$

and

$$
\sum_{n=1}^{\infty} \frac{e^{-\xi n}}{n}=-\ln \left(1-e^{-\xi}\right)
$$

we then obtain the self-energy as

$$
\Sigma(T, L)=m_{0} \frac{\lambda_{0} m_{0}}{2 \pi} \mathcal{F}\left(\frac{m_{0}}{T}, m_{0} L\right),
$$

where

$$
\begin{aligned}
& \mathcal{F}(x, y)=-\frac{\ln \left(1+e^{-x}\right)}{x}-\frac{\ln \left(1+e^{-y}\right)}{y} \\
& +2 F(x, y)-4 F(x, 2 y)-4 F(2 x, y)+8 F(2 x, 2 y),
\end{aligned}
$$


with

$$
F(x, y)=\sum_{n, l=1}^{\infty} \frac{\exp \left(-\sqrt{x^{2} n^{2}+y^{2} l^{2}}\right)}{\sqrt{x^{2} n^{2}+y^{2} l^{2}}}
$$

$T$ - and $L$-dependent coupling constant. - We now consider the four-point function with null external momenta, which defines the $(\beta, L)$-dependent coupling constant. It is given by,

$$
\Gamma_{D}^{(4)}\left(\beta, L ; \lambda_{0}\right) \simeq \lambda_{0}\left[1+\lambda_{0} \Pi(D, \beta, L)\right],
$$

where $\Pi(D, \beta, L)$ is the $(\beta, L)$-dependent one-loop polarization diagram given by

$$
\begin{aligned}
\Pi(D, \beta, L)= & \frac{1}{\beta L} \sum_{n_{1}, n_{2}=-\infty}^{\infty} \\
& \times \int \frac{\mathrm{d}^{D-2} k}{(2 \pi)^{D-2}} \frac{m_{0}^{2}-\left(\mathbf{k}^{2}+\omega_{n_{1}}^{2}+\omega_{n_{2}}^{2}\right)}{\left(\mathbf{k}^{2}+\omega_{n_{1}}^{2}+\omega_{n_{2}}^{2}+m_{0}^{2}\right)^{2}} .
\end{aligned}
$$

Using the dimensionless quantities and subtracting the polar terms, as in the preceding section, the finite polarization reads

$$
\Pi(T, L)=\frac{m_{0}}{2 \pi} \mathcal{G}\left(\frac{m_{0}}{T}, m_{0} L\right),
$$

where the function $\mathcal{G}(x, y)$ is defined by

$$
\begin{aligned}
\mathcal{G}(x, y)= & \frac{\ln \left(1+e^{-x}\right)}{x}-\frac{1}{1+e^{x}}+\frac{\ln \left(1+e^{-y}\right)}{y} \\
& -\frac{1}{1+e^{y}}+2 G(x, y)-4 G(2 x, y) \\
& -4 G(x, 2 y)+8 G(2 x, 2 y),
\end{aligned}
$$

with

$$
G(x, y)=\sum_{n, l=1}^{\infty} \exp \left(-\sqrt{x^{2} n^{2}+y^{2} l^{2}}\right)-F(x, y) .
$$

Then using eq. (20) this provides us with the finite thermal and boundary-dependent coupling constant,

$$
\left.g\left(T, L ; \lambda_{0}\right) \equiv \Gamma_{3}^{(4)}\left(T, L, \lambda_{0}\right) \simeq \lambda_{0}\left[1+\lambda_{0} \Pi(T, L)\right)\right] .
$$

Phase transition. - We now discuss the phase transition occurring in the GN model with a compactified spatial dimension. We start by looking for the existence of an infrared stable fixed point.

Fixed-point structure. An indication of a secondorder transition is obtained from a renormalization group argument. In this case, the existence of an infrared stable fixed point at criticality, can be shown from a study of the infrared behaviour of the beta-function, i.e. in the neighbourhood of vanishing external momentum, $|p| \approx 0$. We consider the thermal boundary-dependent coupling constant at criticality $m=m\left(T_{c}(L)\right)=0$, with an external small momentum $p$, given by

$$
g(m=0 ;|p| \approx 0)=\frac{\lambda_{0}}{\left[1-\lambda_{0} \Pi(m=0 ; p)\right]} .
$$

We first evaluate the one-loop polarization $\Pi(D, T, L, p)$ using a Feynman parameter $x$, and taking the mass parameter as the thermal boundary-dependent mass $m(T, L)$, which vanishes at criticality. We have,

$$
\begin{aligned}
& \Pi(D, T, L, p)=\frac{1}{\beta L} \int_{0}^{1} \mathrm{~d} x \sum_{n_{1}, n_{2}=-\infty}^{\infty} \\
& \times \int \frac{\mathrm{d}^{D-2} k}{(2 \pi)^{D-2}} \frac{m^{2}-\left(\mathbf{k}^{2}+\omega_{n_{1}}^{2}+\omega_{n_{2}}^{2}\right)}{\left(\mathbf{k}^{2}+\omega_{n_{1}}^{2}+\omega_{n_{2}}^{2}+M_{x}^{2}\right)^{2}},
\end{aligned}
$$

where $\quad M_{x}^{2}=M_{x}^{2}(p, T, L, x)=m^{2}(T, L)+p^{2} x(1-x)$. Equation (27) can be rewritten as

$$
\begin{aligned}
& \Pi(D, T, L, p)=\frac{1}{\beta L} \sum_{n_{1}, n_{2}=-\infty}^{\infty} \int_{0}^{1} \mathrm{~d} x \\
& \times \int \frac{\mathrm{d}^{D-2} k}{(2 \pi)^{D-2}} \frac{M_{x}^{2}-\left(\mathbf{k}^{2}+\omega_{n_{1}}^{2}+\omega_{n_{2}}^{2}\right)}{\left(\mathbf{k}^{2}+\omega_{n_{1}}^{2}+\omega_{n_{2}}^{2}+M_{x}^{2}\right)^{2}} \\
& -\frac{p^{2}}{\beta L} \sum_{n_{1}, n_{2}=-\infty}^{\infty} \int_{0}^{1} \mathrm{~d} x \\
& \times \int \frac{\mathrm{d}^{D-2} k}{(2 \pi)^{D-2}} \frac{x(1-x)}{\left(\mathbf{k}^{2}+\omega_{n_{1}}^{2}+\omega_{n_{2}}^{2}+M_{x}^{2}\right)^{2}} .
\end{aligned}
$$

For $|p| \approx 0$, we can neglect the second term in eq. (28). Treating the first term in eq. (28) with the same techniques already used above, specializing for $D=3$, the polarization $\Pi(T, L, p)$ reads,

$$
\Pi(T, L, p)=\int_{0}^{1} \mathrm{~d} x \frac{M_{x}}{2 \pi} \mathcal{G}\left(\frac{M_{x}}{T}, M_{x} L\right) .
$$

At criticality, $m(T, L)=m\left(T_{c}(L)\right)=0$, and for $|p| \sim 0$, keeping up to terms linear in $|p|$, from the above equation we get,

$$
\Pi\left(T_{c}, L,|p| \sim 0\right) \approx A\left(T_{c}, L\right)|p|+B\left(T_{c}, L\right),
$$

with

$$
A\left(T_{c}, L\right)=A=-\frac{1}{16}
$$

and

$$
\begin{aligned}
& B\left(T_{c}, L\right)=\left(\frac{T_{c}}{2 \pi}+\frac{1}{2 \pi L}\right) \ln 2-\mathcal{O}\left(\frac{1}{T_{c}}, L\right) \\
& +2 \mathcal{O}\left(\frac{1}{T_{c}}, 2 L\right)+2 \mathcal{O}\left(\frac{2}{T_{c}}, L\right)-4 \mathcal{O}\left(\frac{2}{T_{c}}, 2 L\right)
\end{aligned}
$$

where

$$
\mathcal{O}(x, y)=\sum_{n, l=1}^{\infty} \frac{1}{\sqrt{x^{2} n^{2}+y^{2} l^{2}}}
$$

and we have used that $\int_{0}^{1} \mathrm{~d} x \sqrt{x(1-x)}=\pi / 8$. 
The coupling constant in eq. (26) has dimension of $|p|^{-1}$. Taking $|p|$ as a running scale, we define a dimensionless coupling constant

$$
g^{\prime}=|p| g=\frac{|p| \lambda_{0}}{1-\lambda_{0}\left[A\left(T_{c}, L\right)|p|+B\left(T_{c}, L\right)\right]}
$$

and the beta-function,

$$
\beta\left(g^{\prime}\right)=|p| \frac{\partial g^{\prime}}{\partial|p|} ;
$$

we find that the condition of a non-trivial infrared stable fixed point is fulfilled by the solution

$$
g_{\star}^{\prime}=-\frac{1}{A}=16 .
$$

Then the infrared stable fixed point is independent of the length of the system and of the free-space coupling constant. Thus, we conclude that the phase transition, while it survives with decreasing $L$, is a second-order one. We now discuss the $L$-dependence of the critical temperature.

Size effects on the critical temperature. Replacing in eq. (18) $\lambda_{0}$ by $g\left(T, L ; \lambda_{0}\right)$ given in eq. (25), we obtain a new self-energy, $\mathcal{S}\left(T, L ; \lambda_{0}\right)$, which incorporates the thermal and boundary corrections to the coupling constant. Then the $(T, L)$-dependent mass is

$$
\begin{aligned}
m(T, L)= & m_{0}+\mathcal{S}\left(T, L ; \lambda_{0}\right) \\
= & m_{0}\left\{1+\frac{\lambda_{0} m_{0}}{2 \pi} \mathcal{F}\left(\frac{m_{0}}{T}, m_{0} L\right)\right. \\
& \left.\times\left[1+\frac{\lambda_{0} m_{0}}{2 \pi} \mathcal{G}\left(\frac{m_{0}}{T}, m_{0} L\right)\right]\right\} .
\end{aligned}
$$

The condition for a phase transition, $m(T, L)=0$, provides a critical surface defined by the critical temperature, the size of the system, $L$, and the the zero-temperature coupling constant in the the absence of boundaries, $\lambda_{0}$. This surface defines $L$-dependent values of the critical temperature, $T_{c}\left(L ; \lambda_{0}\right)$.

The dependence of $T_{c}$ on $\lambda_{0}$ is illustrated in fig. 1, for some values of $L$. In fig. 2, we present the behavior of the critical temperature as the length of the system diminishes, for some values of $\lambda_{0}$. From these plots, we find that there exists a minimal length below which the transition is suppressed.

We see from fig. 1 that the curve for $L \rightarrow \infty$ coincides with the curve obtained in a previous article in the absence of boundaries [23]. Moreover, we find that as the size of the system is diminished, the transition disappears. The suppression of the transition below a minimal size is illustrated in fig. 2 for several values of the fixed coupling constant. These minimal sizes, $L_{\min }$, are characterized by the vanishing of the transition temperature and can be estimated. Take for instance the value $l_{\text {min }}^{-1} \approx 0.826$ corresponding to $\lambda=5.0$ (the dashed curve in the figure). This gives $L_{\min } \approx 1.21 m_{0}^{-1}$. Now, let us take for $m_{0}$, the mass of the Gross-Neveu fermion, to be the effective quark

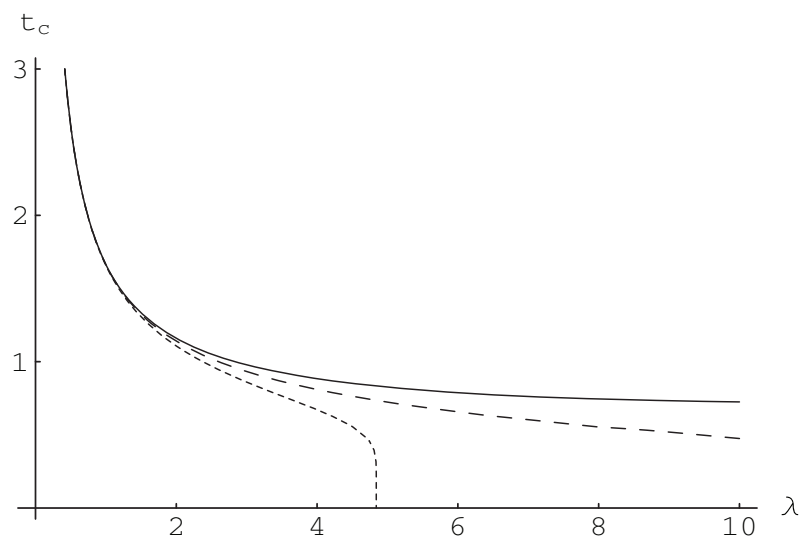

Fig. 1: Critical temperature in units of $m_{0}, t_{c}=T_{c} / m_{0}$, as a function of the free-space coupling constant (in units of $m_{0}^{-1}$ ), $\lambda=m_{0} \lambda_{0} / 2 \pi$, for some values of the compactification length (in units of $m_{0}^{-1}$ ), $l=m_{0} L: \infty, 1.4$ and 1.2 (full, dashed and dotted lines, respectively).

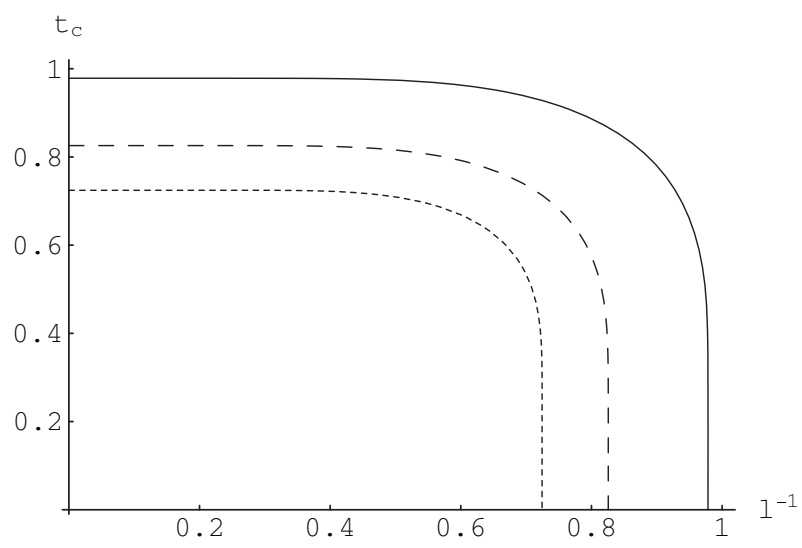

Fig. 2: Critical temperature in units of $m_{0}, t_{c}=T_{c} / m_{0}$, as a function of the inverse of the compactification length (in units of $\left.m_{0}\right), l^{-1}=1 / m_{0} L$, for some values of $\lambda=m_{0} \lambda_{0} / 2 \pi: 3.0,5.0$ and 10.0 (full, dashed and dotted lines, respectively).

mass of the proton [37], $m_{0} \approx 330 \mathrm{MeV}$. We get, using the conversion $1 \mathrm{MeV}^{-1}=197 \mathrm{fm}, L_{\min } \approx 0.72 \mathrm{fm}$. This is of the order of magnitude of the estimated size of a meson, $L_{\text {meson }}$, of $\sim 2 / 3$ of the size of a hadron, that is, $L_{\text {meson }} \sim 0.92 \mathrm{fm}$.

Concluding remarks. - As we have already stated, the GN model may be seen as an effective theory for QCD, with an arbitrary zero-temperature coupling constant in the absence of boundaries. From a non-perturbative analysis of the four-point function, an indication of the existence of a phase transition is obtained, in a similar way as in the linear response theory and in the BCS model of superconductivity. Thereby a critical region can be established and the analysis of the free energy provides the nature of the transition, as being of second order. Also, we have shown that we can define a beta-function, which has a non-trivial infrared fixed point, which reinforces the conclusion that the transition is a second-order one. 
As the central part of this work, we have analyzed effects due to the finite size of the system, with the main conclusion that no transition exists below a minimal size. This size depends on the chosen model, i.e., on the value of the coupling constant we choose. In any case, this is understandable from a physical point of view, since longrange correlations can not persist in small distances. This can be related with the existence of a minimal thickness sustaining superconducting transitions in films [38,39], in the framework of the Ginzburg-Landau model. Moreover, taking for instance the model defined by $\lambda=5.0$, it can be inferred from fig. 2 that, for sizes of the system slightly larger than $L_{\min } \approx 0.72 \mathrm{fm}$ (twice or more the minimum size), of order of magnitude of the estimated size of a meson, the transition temperature is $\sim 272 \mathrm{MeV}$, which is compatible with the deconfining hadronic temperature. Similar results are obtained for other values of $\lambda$.

The results in this note have been possible by employing the massive GN model in three dimensions. In previous works devoted to get insights into the behaviour of hadronic matter, the massless GN model, in its version with a large number of components, has been employed often. Using the one-component massive GN model in three dimensions and taking the fermion mass as a physical parameter (the effective quark mass), we have been able, using analytical means, to study finite-size effects on the transition. A particularly interesting aspect of the results is that the minimal sizes allowed and critical temperatures are compatible with characteristic quantities in particle physics, e.g., the size of a meson and the deconfining hadronic temperature. This gives us confidence in the results derived from the model. A rigorous study involving additional aspects of this transition, including the determination of critical exponents, is left for future work.

\section{$* * *$}

This work was partially supported by CNPq, CAPES and FAPERJ (Brazil) and NSERC (Canada). We thank kind hospitality from The Theoretical Physics Institute, Department of Physics, University of Alberta.

\section{REFERENCES}

[1] Gross D. J. and Neveu A., Phys. Rev. D, 10 (1974) 3235 .

[2] Barducci A., Casalbuoni R., Modugno M., Pettini G. and Gatto R., Phys. Rev. D, 51 (1995) 3042.

[3] Zhou B. R., Commun. Theor. Phys., 32 (1999) 425.

[4] Brzoska A. and Thies M., Phys. Rev. D, 65 (2002) 125001.

[5] Kneur J.-L., Pinto M. B., Ramos R. O. and Staudt E., Phys. Rev. D, 76 (2007) 045020.

[6] Boenmer C., Fritsch U., Kraus S. and Thies M., Phys. Rev. D, 78 (2008) 065043.

[7] Höfling F., Nowak C. and Wetterich C., Phys. Rev. $B, 66$ (2002) 205111.
[8] Malbouisson A. P. C., Malbouisson J. M. C., Santana A. E. and Silva J. C., Phys. Lett. B, 583 (2004) 373.

[9] Khanna F. C., Malbouisson A. P. C., Malbouisson J. M. C., Queiroz H., Rocha-Filho T. M., Santana A. E. and Silva J. C., Phys. Lett. B, 624 (2005) 316.

[10] Kohyama H., Phys. Rev. D, 77 (2008) 045016.

[11] Feinberg J., Int. J. Mod. Phys., 17 (2002) 898.

[12] Semenoff G. W., Phys. Rev. Lett., 53 (1984) 2449.

[13] Gusynin V. P., Sharapov S. G. and Carbotte J. P., Int. J. Mod. Phys. B, 21 (2007) 4611.

[14] Khveshchenko D. V., Phys. Rev. Lett., 87 (2001) 246802.

[15] Semenoff G. W. and Wijewardhana L. C. R., Phys. Rev. D, 45 (1992) 1342.

[16] Juričić V., Herbut I. F. and Semenoff G. W., Phys. Rev. B, 80 (2009) 081405(R).

[17] Herbut I. F., Phys. Rev. Lett., 97 (2006) 146401.

[18] Herbut I. F., Juričić V. and Roy B., Phys. Rev. B, 79 (2009) 085116.

[19] Jackiw R. and Pi S.-Y., Phys. Rev. Lett., 98 (2007) 266402.

[20] Novoselov K., Nat. Mater., 6 (2007) 720.

[21] Thies M., Phys. Rev. D, 69 (2004) 067703.

[22] Fushchich W. I. and Zhdanov R. Z., Phys. Rep., 172 (1989) 123.

[23] Khanna F. C., Malbouisson A. P. C., Malbouisson J. M. C. and Santana A. E., EPL, 92 (2010) 11001.

[24] Khanna F. C., Malbouisson A. P. C., Malbouisson J. M. C. and SAntanA A. E., Thermal Quantum Field Theory: Algebraic Aspects and Applications (World Scientific, Singapore) 2009.

[25] Malbouisson A. P. C., Malbouisson J. M. C. and Santana A. E., Nucl. Phys. B, 631 (2002) 83.

[26] Abreu L. M., Malbouisson A. P. C., Malbouisson J. M. C. and Santana A. E., Nucl. Phys. B, 819 (2009) 127.

[27] Abreu L. M., Malbouisson A. P. C. and Malbouisson J. M. C., EPL, 90 (2010) 11001.

[28] Abreu L. M., Malbouisson A. P. C. and Malbouisson J. M. C., Phys. Rev. D, 83 (2011) 025001.

[29] Zhu X. Q., Khanna F. C. and Umezawa H., Phys. Rev. C, 43 (1991) 2891.

[30] de Calan C., Faria da Veiga P. A., Magnen J. and SÉneor R., Phys. Rev. Lett., 66 (1991) 3233.

[31] Gawedzki K. and Kupiainen A., Phys. Rev. Lett., 55 (1985) 363.

[32] Gawedzki K. and Kupiainen A., Nucl. Phys. B, 262 (1985) 33.

[33] Parisi G., Nucl. Phys. B, 100 (1975) 368.

[34] Doniach S. and Sondheimer E. H., Green's Functions for Solid State Physicists (W.A. Benjamin, London) 1974.

[35] Kirzhnits D. A. and Linde A. D., Ann. Phys. (N.Y.), 101 (1976) 195.

[36] Elizalde E., Ten physical applications of spectral zetafunctions. Lect. Notes Phys., Vol. 35 (Springer-Verlag, Berlin) 1995.

[37] Maltman K. and Isgur N., Phys. Rev. D, 29 (1984) 952.

[38] Abreu L. M., de Calan C., Malbouisson A. P. C., Malbouisson J. M. C. and Santana A. E., J. Math. Phys., 46 (2005) 012304.

[39] Malbouisson A. P. C., Malbouisson J. M. C. and Pereira R. C., J. Math. Phys., 50 (2009) 083304. 